\title{
Laparoscopic Ladd's Procedure: Treatment of Choice for Midgut Malrotation in Infants and Children
}

\author{
Amy B. Stanfill, MD, Richard H. Pearl, MD, Kavitha Kalvakuri, MBBS, \\ Lizabeth J. Wallace, MS, and Ravindra K. Vegunta, MBBS
}

\begin{abstract}
Introduction: Rotational anomalies of the midgut encompass a broad spectrum of incomplete rotational events with malfixation of the intestines during fetal development. Ladd's procedure, as a correction of these anomalies, has traditionally been performed by laparotomy. In our institution, the laparoscopic Ladd's (LL) procedure was introduced in May 2004 and soon became the standard approach.

Materials and Methods: A retrospective analysis of all Ladd's procedures in children in our institution between September 1998 and June 2008 was performed. Outcomes between the open (OL) and LL procedures were compared.

Results: A total of 156 children underwent Ladd's procedure during the study period. There were 120 open and 36 laparoscopic procedures. Overall, $75 \%$ of patients in each group were symptomatic, most commonly with emesis and pain. Duration of surgery was similar in both groups. Time to starting feeds, and amount of time to attain full feeding, was significantly less in the LL group. Postoperative length of stay was significantly less in the patients having LL. Conversion rate to OL from LL was $8.3 \%$.

Conclusions: LL can be performed safely in selected patients with no increase in complications. Short-term results are superior to OL and can be achieved without any increase in operative duration.
\end{abstract}

\section{Introduction}

$\mathbf{T}$ HE TERM "MALROTATION" encompasses a broad spectrum of intestinal rotation and fixation anomalies. The primitive intestinal tract begins to form at approximately 4 weeks of gestation, and at 10 weeks of gestation, the midgut undergoes a 270-degree counterclockwise rotation around the axis of the superior mesenteric artery (SMA). By week 12, the midgut becomes attached to the posterior abdominal wall via multiple peritoneal reflections providing broad-based stabilization from the ligament of Treitz to the cecum. Variations in this sequence of rotation and fixation of the midgut result in malrotation with a narrowed vascular pedicle, predisposing to torsion or volvulus. Intestinal obstruction and ischemia are the consequences of volvulus, with a potential for necrosis of the entire midgut. Death or morbidity of short-gut syndrome can ensue. Nonischemic obstruction of the duodenum can also occur as a result of Ladd's bands compressing and causing partial obstruction of the duodenum. ${ }^{1}$

In the newborn, bilious emesis is the most frequent presenting symptom, reported in $71-100 \%$ of patients. ${ }^{2,3}$ In infants and children older than 2 months, bilious and nonbilous emesis occur at equal rates (49\%). However, abdominal pain, failure to thrive, diarrhea, or constipation are also noted. In a recent series, $20 \%$ of patients were diagnosed with malrotation while undergoing exploratory laparotomy for an associated anomaly. ${ }^{3}$ The most feared consequence of malrotation is midgut volvulus, a true surgical emergency. In patients with symptomatic midgut volvulus, $25 \%$ occur in the first week of life and $56 \%$ occur before 1 month of age. In these children, bilious (green) vomiting is the most common presenting symptom. 4

Traditional correction of malrotation is by laparotomy and Ladd's procedure, as first performed by William Ladd, the First Chief of Surgery at Boston Children's Hospital. ${ }^{5}$ The first report of laparoscopic evaluation and correction of malrotation was in 1995, ${ }^{6}$ and similar reports followed shortly thereafter by Gross and Waldhausen, from separate institutions. ${ }^{7,8}$ Comparison of OL and LL procedures have shown that LL is safe and effective in adults. ${ }^{9}$ Several small series, with 7-12 patients each, have demonstrated the safety and efficacy of LL in infants and children. ${ }^{10-12}$ Despite this, LL is still not routinely performed as the procedure of choice for rotational anomalies in infants and children. In our institution,

Department of Pediatric Surgery, University of Illinois College of Medicine at Peoria, Children's Hospital of Illinois, Peoria, Illinois. 
LL was introduced in 2004 and quickly became the preferred method for correction of malrotation. In this article, we document the safety and effectiveness of LL procedure in children and infants on a larger scale.

\section{Materials and Methods}

A retrospective analysis of all Ladd's procedures performed in our institution between September 1998 and June 2008 was undertaken. Institutional review board approval was obtained for this analysis. LL was introduced in our institution in May 2004 and has since become the preferred approach. Newborns undergoing surgery for congenital diaphragmatic hernia, gastroschisis, or omphalocele were excluded from the analysis. Records were reviewed for patient characteristics (i.e., age, weight, and concomitant congenital anomalies), findings at operative exploration (i.e., presence or absence of volvulus, need for enterectomy, and duration of surgery), postoperative data (i.e., time to start of feeds, time to attain full feeding, and length of stay), complications (i.e., wound infection, readmission, bowel obstruction after Ladd's, volvulus after Ladd's, and incisional hernia), and length of follow-up. All procedures were performed by a pediatric surgeon assisted by a general surgery resident.

\section{Diagnosis}

Diagnosis of rotational anomaly was made most commonly by upper gastrointestinal (UGI) contrast examination. Contrast enema or computed tomography (CT) scan suggested the diagnosis in a few cases. In all cases, the operating surgeon conferred directly with the radiologist in review of the images and, in some cases, was present during the radiologic evaluation. If diagnosis of malrotation was entertained during the radiographic evaluation, but diagnosis remained ambiguous, laparoscopy was used to confirm the presence or absence of a rotational anomaly.

\section{Technique}

OL was performed through an upper abdominal transverse incision. In LL, the peritoneal cavity was accessed initially via the umbilicus with a 3- or 5-mm disposable trocar (US Surgical, Norwalk, CT). After insufflation and under direct visualization, two other working ports were placed in the right- and leftlower abdomen. If needed for retraction, one additional port was placed in the epigastrum. After gaining access to the abdomen, the operative procedure in both the OL and LL was equivalent. Abdominal exploration was performed to confirm the diagnosis of rotational anomaly. The ligament of Treitz was identified by elevating the transverse colon and the cecum located by gross inspection. If the ligament Treitz was not properly positioned, a diagnosis of malrotation was made and a Ladd's procedure performed. The Ladd's bands were incised, the duodenum mobilized, straightened, and placed in the right abdomen coursing caudally. The mesentery of the small bowel was examined and broadened by incising the furled portions of the anterior leaflet of the mesentery, taking care not to injure the vessels within. The cecum was identified and the appendix removed, via intra- or extracorporeal appendectomy, at the operating surgeon's discretion. The colon was then placed in the left abdomen.

\section{Results}

A total of 156 children underwent Ladd's procedure during the study period. Of these, 120 were open procedures and 36 were laparoscopic. Follow-up ranged from 9 months to 10 years. Overall, $75 \%$ of the patients in each group presented with symptoms, the most common being emesis and abdominal pain. There were some significant differences in our patient demographics. Median age in the OL group was 3 months versus 7 months in the LL group $(P=0.004)$ and median weight was $4 \mathrm{~kg}$ in the OL group versus $7.2 \mathrm{~kg}$ in the LL group $(P=0.002)$. A significantly larger proportion of patients in the OL group had concomitant congenital heart disease, as compared with the LL group (21.7 versus $5.6 \%$; $P=0.027)$. However, eliminating the patients with congenital heart disease from the analysis did not change the outcomes significantly.

Intraoperative findings did not differ significantly between the two groups. Although a larger percentage of children in the OL group had a volvulus noted at exploration than in the LL group (16.7 versus $11.1 \% ; P=0.599)$, this difference was not significant. Of 120 patients in the OL group, 8 required bowel resection $(7.7 \%)$, whereas no patients in the LL group required a bowel resection; this did not reach statistical significance $(P=0.199)$. Median duration of surgery was similar between the two groups (76 minutes in OL versus 75 in LL; $P=0.22)$. The rate of conversion to open in the LL group was $8.3 \%$. These occurred in case numbers 8, 18, and 19 in this series, with no conversions in the last 17 consecutive cases. All cases were converted due to inadequate visualization of key structures. Appendectomy was performed concomitantly in $97 \%$ of the procedures. The appendix was left intact, if the patient had any evidence of neurogenic bowel or bladder and might require use of the appendix as a conduit in the future.

Postoperative data displayed several significant differences between the two groups (Table 1). The median number of days to starting feeds and achieving full feeds was significantly higher in the OL, compared to the LL, group (4 days in OL versus 1 in LL; $P=0.001$, and 6 days in OL versus 2 in LL; $P=0.001$, respectively). Length of postoperative stay was also significantly longer in the OL group (7 days in OL versus 3 in LL; $P=0.001)$. Notable differences were also seen in the rates of postoperative complications (Table 2). Median follow-up for the OL group was 5.7 years, compared to 2.4 in the LL group. Wound infections occurred significantly more often in the OL group (11.7 in OL versus $0 \%$ in $L L ; P=0.041)$. In the OL group, there were 33 readmissions $(27.5 \%)$ during the follow-up period, compared to only $4(11.1 \%)$ in the LL group $(P=0.046)$. Postoperative bowel obstruction was noted in 16 of the 120 patients in the OL group (13.3\%). To date, no postoperative bowel obstruction has occurred in the patients undergoing $\operatorname{LL}(P=0.024)$. Incisional hernia rates of 5 and

Table 1. Postoperative Feeding and Length of Stay

\begin{tabular}{lccc}
\hline & OL & LL & P-value \\
\hline Days to start feeds & 4 & 1 & 0.001 \\
Days to full feeds & 6 & 2 & 0.001 \\
Postoperative LOS & 7 & 3 & 0.001 \\
\hline
\end{tabular}

OL, open laparoscopic procedure; LL, laparoscopic Ladd's procedure; LOS, length of stay. 
Table 2. Postoperative Complications

\begin{tabular}{lccc}
\hline Complication & OL (\%) & LL (\%) & P-value \\
\hline Wound infection & 11.7 & 0 & 0.041 \\
Readmission & 27.5 & 11.1 & 0.046 \\
Postoperative bowel obstruction & 13.3 & 0 & 0.024 \\
Postoperative volvulus & 0.8 & 5.6 & 0.133 \\
Incisional hernia & 5 & 0 & 0.337 \\
\hline
\end{tabular}

OL, open laparoscopic procedure; LL, laparoscopic Ladd's procedure.

$0 \%$ were recorded in OL versus $L L$, respectively $(P=0.337)$. Volvulus after Ladd's was less common in the OL group, but did not reach statistical significance (0.8 versus $5.6 \%$; $P=0.133$ ). None of the children with volvulus after OL or LL required enterectomy.

\section{Discussion}

It is widely accepted that a diagnosis of malrotation mandates correction of the abnormality by Ladd's procedure. The optimal method of performing Ladd's procedure is currently being debated. This report of 36 patients undergoing laparoscopy for diagnosis and definitive treatment of malrotation represents the largest series available for review in the literature. The data presented in this article support the findings of several smaller series reported previously, which support LL as safe and efficacious in infants and children. ${ }^{10-12}$ Most striking in our series was the significant drop in the time to start and attain full feeding in the LL patient population. Our findings are similar to previously reported data for LL, in which most patients were started on feedings on postoperative day 1 and advanced to goal feedings in the ensuing 24 hours. ${ }^{10}$ Early feeding was a significant factor that led to decreased length of stay, as seen in other series, as well (range, 2-3.6 days). ${ }^{10-12}$ Additionally, the significant decrease in the rate of wound infection and incisional hernia after LL must be considered, as these can be a source of significant morbidity requiring wound care and the possibility of further surgery and expense.

In Gross' original series of 156 patients, reported in 1951, the rate of recurrent volvulus after Ladd's operation was
$0.6 \%{ }^{4}$ This finding is similar to the current OL group $(0.8 \%)$. The higher percentage of patients in the LL group with recurrent volvulus (5.6\%) may be due to decreased adhesion formation from the laparoscopic surgical approach. The researchers acknowledge the potential increased risk of volvulus after LL, when compared to OL, and take this risk very seriously. However, the laparoscopic approach did provide significant protection against postoperative bowel obstruction, noted in 16 of the 120 patients in the OL group $(13.3 \%)$. This is similar to the reported incidence of $14 \%$ in elective open Ladd's procedure in the setting of complex congenital heart disease. ${ }^{13}$ Of note, postoperative bowel obstruction in the OL group (13.3 in OL versus $0 \%$ in LL) occurred at twice the rate of postoperative volvulus in the LL group (0.8 in OL versus $5.6 \%$ in LL). This is an important consideration when evaluating the risk versus benefit comparison of the two procedures. We would suggest each institution analyze the data critically, discuss the issues with the family prior to surgery, and, together, make an informed decision on an individual patient basis.

Our median operative duration of 75 minutes is well within the reported range for LL procedures (average, 58 minutes ${ }^{10}$ to 120 minutes ${ }^{12}$ ). In previous reports from our center, documenting a learning curve for other laparoscopic procedures, we would anticipate operative times to decrease. ${ }^{14,15}$ Our study was limited by several factors. The sample-size disparity between groups was noted, but as we continue to perform a larger number of LLs, this discrepancy will be alleviated (Fig. 1). In our early experience in performing LL, the patients were significantly older and weighed more. However, if the patient-characteristic data are inspected sequentially, the age and weight of our LL patients decreased as time passed. We believe this represents progression along a learning curve, and an improvement in surgeon technique, in performing the procedure in younger and smaller infants. With this in mind, we also expect our rate of conversion from LL to OL (3 of 36 patients; $8.3 \%$ ) to decrease over time. In our experience, the main limitation in performing this procedure in an infant is often the combination of bowel distension and a smaller intra-abdominal domain, leading to inadequate working space within the abdomen. We strongly believe that once access to the abdomen is gained, the operative procedure (LL versus OL) performed should be equivalent. If sufficient

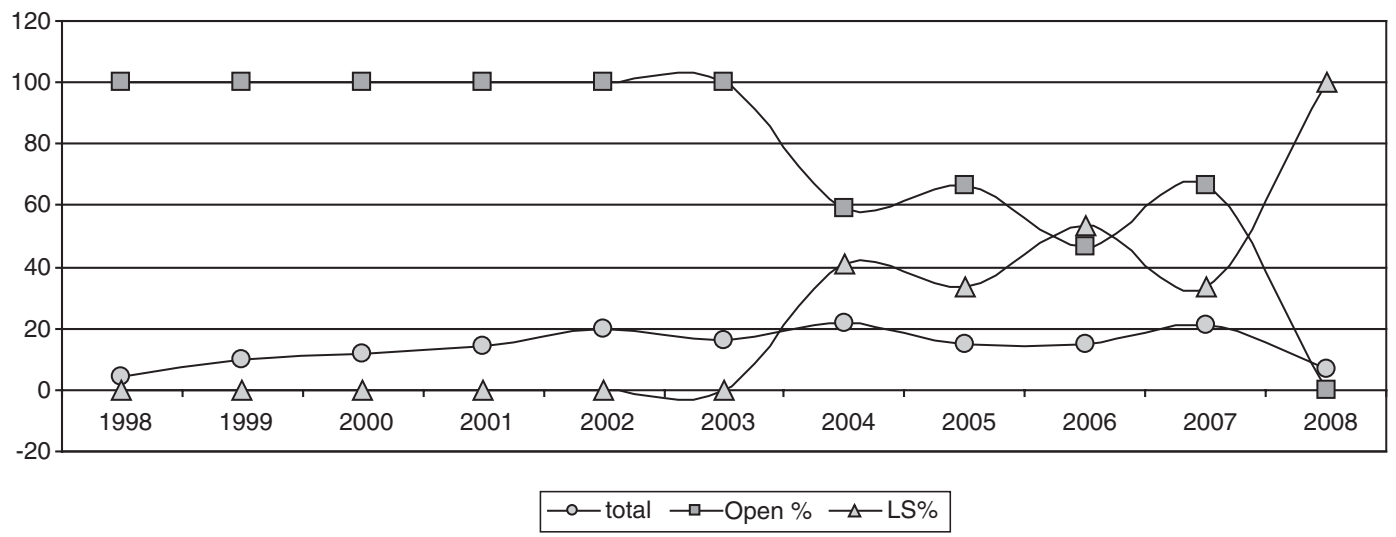

FIG. 1. Ladd's procedures ordered consecutively. Total number of Ladd's procedures noted by circles, percentage of OL noted by squares, and percentage of LL noted by triangles. The 2008 data only represent data from January to June 2008. 
working space cannot be achieved, and key structures (the position of the ligament of Treitz) not appropriately visualized, the operation is converted to open. We recommend this policy across all patient age groups and sizes.

\section{Conclusions}

All of the benefits of LL can be obtained without increases in operative duration or complications. We believe that laparoscopic diagnosis and treatment of intestinal malrotation is feasible, safe and should be considered as the treatment of choice for rotational anomalies in infants and children.

\section{Disclosure Statement}

No competing financial interests exist.

\section{References}

1. Akin JT. The small intestines. In: Gray SW, Skandalakis JE (eds): Embryology for Surgeons. Philadelphia: WB Saunders, 1972, pp. 129-141.

2. Adrasssy RJ, Mahour H. Malrotation of the midgut in infants and children: A 25-year review. Arch Surg 1981;116: 158-160.

3. Powell KM, Othersen HB, Smith CD. Malrotation of the intestines in children: The effect of age on presentation and therapy. J Pediatr Surg 1989;24:777-780.

4. Gross RE. Malrotation of the intestine and colon. In: Gross RE (ed): The Surgery of Infancy and Childhood. Philadelphia: WB Saunders, 1953, pp. 192-203.

5. Ladd WE, Gross RE. Intestinal obstruction resulting from malrotation of the intestines and colon. In: Ladd WE, Gross RE (eds): Abdominal Surgery of Infancy and Childhood. Philadelphia: WB Saunders, 1941, pp. 53-70.

6. van der Zee DC, Bax NM. Laparoscopic repair of acute volvulus in a neonate with malrotation. Surg Endosc 1995; 9:1123-1124.
7. Gross E, Chen MK, Lobe TE. Laparoscopic evaluation and treatment of intestinal malrotation in infants. Surg Endosc 1996;10:936-937.

8. Waldhausen JH, Sawin RS. Laparoscopic Ladd's procedure and assessment of malrotation. J Laparoendosc Surg 1996; 6(Suppl):S103-S105.

9. Matzke GM, Dozois EJ, Larson DW, Moir CR. Surgical management of intestinal malrotation in adults: Comparative results for open and laparoscopic Ladd's procedures. Surg Endosc 2005;19:1416-1419.

10. Bass KD, Rothenberg SS, Chang JHT. Laparoscopic Ladd's procedure in infants with malrotation. J Pediatr Surg 1998; 33:279-281.

11. Draus JM, Foley DS, Bond SJ. Laparoscopic Ladd's procedure: A minimally invasive approach to malrotation without midgut volvulus. Am Surg 2007;73;693-696.

12. Mazziotti MV, Strasberg SM, Langer JC. Intestinal rotation abnormalities without volvulus: The role of laparoscopy. J Am Coll Surg 1997;1855;172-176.

13. Tashjian DB, Weeks B, Brueckner M, Touloukian RJ. Outcomes after Ladd's procedure for intestinal malrotation with heterotaxia. J Pediatr Surg 2007;42;528-531.

14. Vegunta RK, Woodland JH, Rawlings RL, Wallace LJ, Pearl RH. Practice makes perfect: Progressive improvement of laparoscopic pyloromyotomy results with experience. J Laparoendosc Adv Surg Tech 2008;18:152-156.

15. Vegunta RK, Ali A, Wallace LJ, Switzer DM, Pearl RH. Laparoscopic appendectomy in children: Technically and safe in all stages of acute appendicitis. Am Surg 2004;70:198-202.

Address correspondence to: Amy B. Stanfill, $M D$ Department of Pediatric Surgery University of Illinois College of Medicine at Peoria Children's Hospital of Illinois 420 Northeast Glen Oak Avenue, Suite 201 Peoria, IL 61603

E-mail: amy.b.stanfill@osfhealthcare.org 